\title{
Mécanique des systémes précontraints appliquée à la structure du cytosquelette
}

\author{
Patrick Cañadas ${ }^{\mathrm{a}}$, Bernard Maurin et René Motro \\ Université Montpellier 2 - Laboratoire de Mécanique et Génie Civil (LMGC) UMR 5508 CNRS - Place Eugène Bataillon, \\ CC048, 34095 Montpellier, France
}

Reçu le 20 avril 2009

\begin{abstract}
Résumé - Ces travaux montrent la pertinence et l'utilité de la modélisation du cytosquelette des cellules par systèmes mécaniques précontraints. Ces recherches s'inscrivent dans le champ de la biomécanique cellulaire et tissulaire et deux applications sont proposées. On présente tout d'abord la recherche de forme d'une cellule isolée par modèles de tenségrité non réguliers, puis on propose une combinaison entre les modèles granulaires et l'analogie de tenségrité. L'extension prospective aux assemblages multicellulaires avec notion d'autocontrainte partagée, par modèles de grilles de tenségrité est ensuite présentée.
\end{abstract}

Mots clés : Cytosquelette / structures de tenségrité / milieux granulaires / modélisation / tissu

Abstract - Prestressed system mechanics applied to the cytoskeleton structure. These works show the pertinence and usefulness in modelling the cytoskeleton by mechanical prestressed systems. They are in the field of cellular and tissular biomechanics and two applications are proposed. We present first the form-finding of an isolated cell by non regular tensegrity systems, and then we propose to combine granular models and tensegrity analogy. A prospective extension to multicellular assemblies considering the concept of shared self-stresses, by the way of tensegrity grids, is subsequently presented.

Key words: Cytoskeleton / tensegrity structures / granular media / modelling / tissue

\section{Introduction}

L'un des enjeux majeurs de la biomécanique cellulaire est de contribuer à la compréhension de processus physiologiques et pathologiques à l'échelle tissulaire ou de l'organe tels que le remodelage osseux, la réparation tissulaire, la réponse immunitaire ou encore le développement de métastases. Il est nécessaire pour cela d'expliciter le lien existant entre le comportement mécanique des cellules adhérentes en interaction avec leur environnement (matrice extracellulaire et/ou cellules avoisinantes) et leurs fonctions biologiques telles que la différentiation, l'expression génique, l'apoptose ou la migration [1].

Depuis une trentaine d'années, de nombreuses études expérimentales ont été conduites pour caractériser la réponse des cellules adhérentes sous des sollicitations mécaniques et traitements biochimiques divers (voir par exemple [2-4]). Cependant, l'hétérogénéité et le manque de cohérence qualitative et quantitative des résultats obtenus a mis en évidence la nécessité de développer

\footnotetext{
a Auteur pour correspondance :
}

canadas@lmgc . univ-montp2.fr un modèle mécanique «général » de cellule afin d'être à même de simuler numériquement les contraintes et déformations appliquées pour évaluer les processus de mécanotransduction associés. La réalisation de cet objectif repose néanmoins sur la capacité à modéliser le comportement mécanique du cytosquelette, support de ces stimuli et de leur transduction en une cascade de signaux et réactions biochimiques intracellulaires. Celui-ci se compose de trois types de biopolymères filamenteux (filaments d'actine, microtubules et filaments intermédiaires) interconnectés entre eux, constituant un lien physique continu entre le noyau et des complexes moléculaires transmembranaires qui permettent l'adhésion de la cellule à son environnement (ex : intégrines, cadhérines, etc.).

Tant que la cellule est en suspension et n'adhère ni à une matrice extracellulaire ni à une cellule voisine, elle prend une forme sphérique avec un cytosquelette ressemblant alors à un gel viscoélastique. C'est au cours du processus d'adhésion et d'étalement de la cellule à son substrat que les filaments du cytosquelette se polymérisent et s'assemblent pour constituer une structure tridimensionnelle précontrainte à l'architecture complexe et dynamique (évolutive dans le temps). Les filaments d'actine 
peuvent aussi s'associer aux molécules de myosine pour générer activement une tension au sein du cytosquelette. En outre, ils se structurent diversement dans la cellule, depuis un fin réseau de courts $(\sim 10 / 100 \mathrm{~nm})$ filaments faiblement tendus au niveau de la membrane cytoplasmique jusqu'à des associations sous forme de grandes $(\sim 10 \mu \mathrm{m})$ fibres fortement tendues reliant des zones d'adhésion éloignées. Les microtubules, quant à eux, sont principalement organisés sous forme étoilée, depuis le centrosome près du noyau jusqu'aux points d'adhésion transmembranaires, et semblent être le support des efforts de compression, possiblement aidé par des filaments intermédiaires agissant comme des haubans passifs. Le cytosquelette apparaît ainsi comme une structure précontrainte en équilibre mécanique avec l'environnement cellulaire [5-7].

Parmi les modèles de cytosquelette développés à ce jour, ceux basés sur l'analogie avec les systèmes de tenségrité (structures spatiales réticulées autocontraintes, composées d'un réseau discontinu de barres comprimées par un réseau continu de câbles tendus, assurant ainsi l'équilibre mécanique de l'ensemble) ont largement démontré leur pertinence par leur capacité à considérer simultanément l'aspect structural tridimensionnel et l'adhésion discrète du cytosquelette, son comportement mécanique global non linéaire (processus de rigidification à la contrainte), le rôle spécifique des filaments d'actine (câbles) et des microtubules (barres), l'effet du changement de tension interne sur la rigidité de l'ensemble, ainsi que la dépendance des propriétés viscoélastiques structurales en fonction de la fréquence de sollicitation [8-18]. Malgré leur pertinence avérée au regard des observations expérimentales, y compris d'un point de vue quantitatif, ces modèles restent néanmoins et à ce jour passifs (la tension dans les câbles ne résulte pas d'un phénomène actif et ne prend donc pas en compte les moteurs moléculaires) et statiques (non prise en considération de processus dynamiques tels que la polymérisation de filaments ou l'évolution de leur connectivité). En outre, ils occultent systématiquement les interactions entre le cytosquelette et la membrane cytoplasmique et ne décrivent qu'assez sommairement l'architecture du cytosquelette car ils se fondent sur une simple analogie câbles/structures d'actine et barres/microtubules, ceci avec un faible nombre de composants (de 6 à 12 barres et 20 à 40 câbles) et des géométries très simplifiées ne permettant pas une bonne corrélation avec la réalité des configurations observées.

\section{Systèmes de tenségrité bio-réalistes}

Afin de répondre à ce problème de bio-fidélité architecturale, une étude basée sur la méthode de recherche de forme par relaxation dynamique a permis de générer des systèmes de tenségrité complexes [19]. On peut ainsi, en prenant comme base morphologique un système à quatre barres, augmenter progressivement le nombre de barres et de câbles, en conservant les propriétés mécaniques et morphologiques associées à l'état de tenségrité : autocontrainte, équilibre, association d'un réseau discontinu de composants comprimés avec un réseau continu d'éléments tendus. Combinée avec des stratégies de contrôle des topologies et des formes ainsi générées, cette approche a été implémentée dans une interface spécifique qui permet de créer un spectre large de systèmes bio-réalistes [20].

On peut l'illustrer par le cas d'une cellule de type épithéliale, caractérisée par un réseau étoilé de microtubules dont le point source est le centrosome situé prés du centre. Elle possède aussi un réseau d'actine corticale en périphérie et d'actine diffuse dans le cytoplasme. Deux systèmes de tenségrité complexes respectant ces observations sont présentés sur la figure 1. On distingue leur caractère dissymétrique et non régulier. Le premier (haut) possède 35 nœuds et 127 éléments tandis qu'on dénombre 41 nœuds et 143 éléments pour le second (bas). Les niveaux de couleur traduisent l'intensité des efforts internes de traction (bleu) et de compression (rouge).

\section{Approche par tenségrité granulaire}

La démarche présentée ci-avant nécessite néanmoins d'être alimentée en informations, issues d'observations expérimentales et permettant de guider l'évolution des modèles vers des architectures réalistes du cytosquelette. Elle a donc conduit au développement d'une autre modélisation, plus originale, qui permet de s'affranchir des problèmes liés à la spécificité de chaque cellule, et, pour aller encore plus loin, de prendre en considération des processus dynamiques tels que la polymérisation/dépolymérisation des filaments du cytosquelette ainsi que leur connectivité variable au cours du temps.

Pour cela, un couplage entre les approches en tenségrité cellulaire et les outils théoriques et numériques spécifiques à la mécanique des milieux discrets a été proposé; ces derniers permettant de modéliser des processus d'interaction dynamique. L'idée consiste à déterminer un modèle pour lequel la géométrie de la structure n'est pas choisie a priori, mais sera issue d'une simulation du processus de structuration du cytosquelette. Puisque celui-ci n'est véritablement polymérisé et assemblé par la cellule qu'au cours de son adhésion et son étalement sur un substrat, la démarche consiste donc à simuler ces deux processus biologiques : c'est donc le modèle qui, en simulant adhésion et étalement, devra structurer (ou plutôt calculer) «son » cytosquelette et non plus le modélisateur. On peut noter, à cet égard, que des travaux utilisant les milieux discrets granulaires ont déjà montré la pertinence d'une telle démarche [21].

Dans cette approche, la cellule et son squelette sont modélisés par un ensemble tridimensionnel de sphères ou grains (Fig. 2) : une grosse sphère centrale (en vert) représente le noyau, les grains en jaune modélisent les protéines intracellulaires connectant les filaments cytosquelettiques et les grains périphériques en violet représentent des molécules membranaires. Certains de ces grains périphériques, répartis aléatoirement, modélisent 

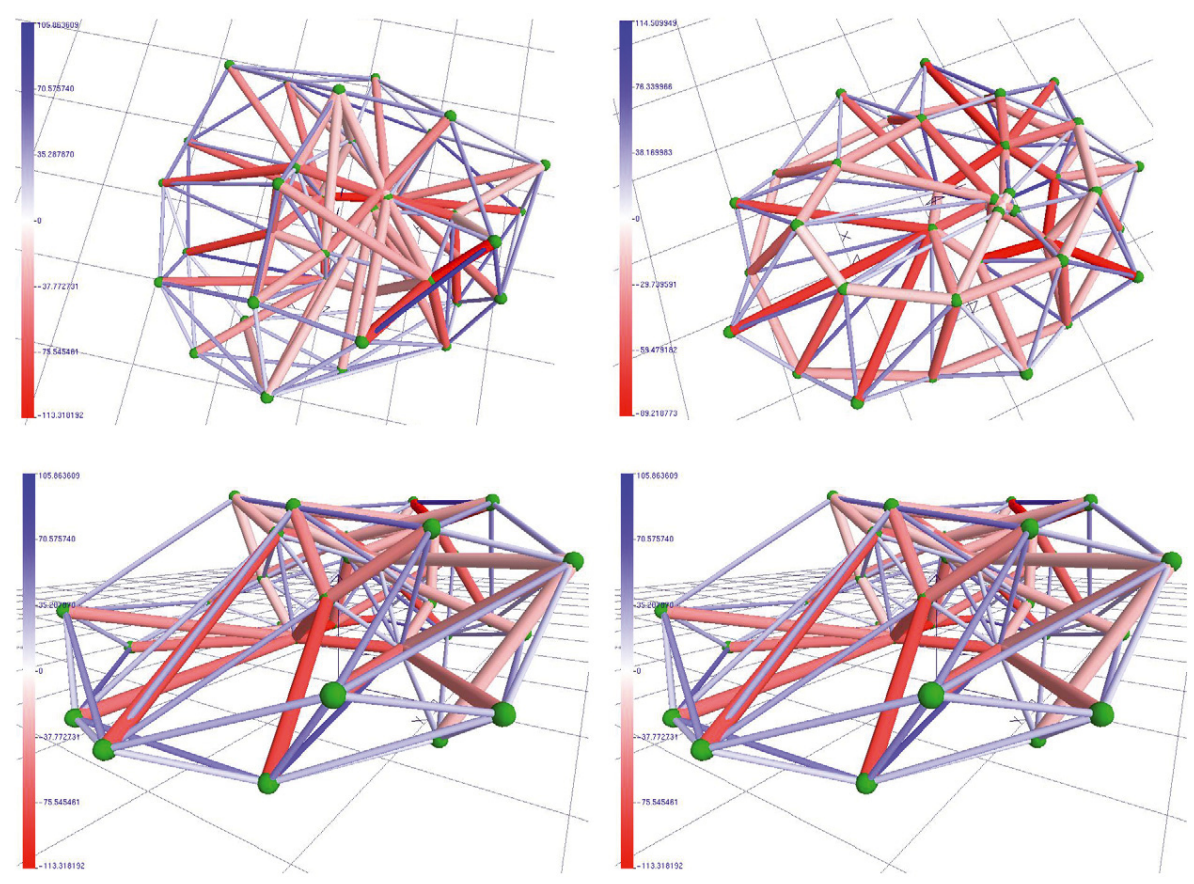

Fig. 1. Deux modélisations d'une cellule épithéliale.
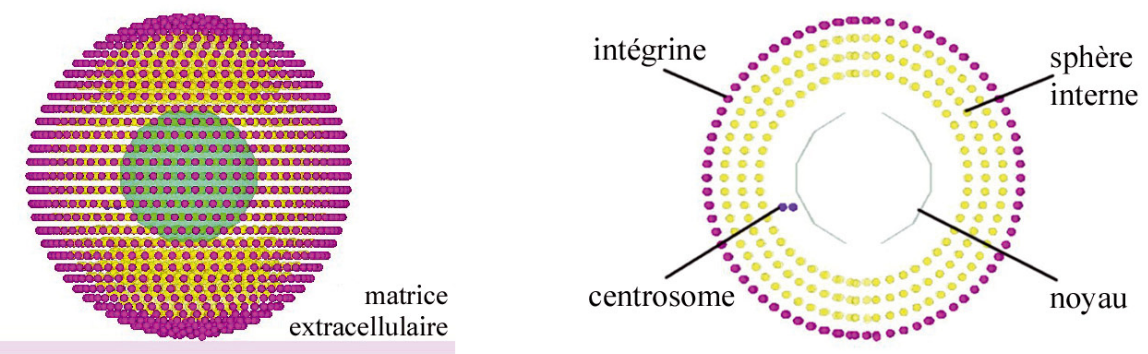

Fig. 2. Modèle de tenségrité «granulaire » (a) vue 3D, (b) coupe méridionale.

les complexes moléculaires d'adhésion (intégrines) tandis qu'une paire de petites sphères, située près du noyau, représente le centrosome. Entre les grains, il existe des interactions mécaniques qui vont permettre de simuler les filaments du cytosquelette. Enfin, pour se rapprocher de la forme réelle d'une cellule non adhérente, l'ensemble des grains est réparti suivant une géométrie globale sphérique.

Les travaux publiés à ce jour concernant la structuration du cytosquelette au cours de l'adhésion et de l'étalement cellulaire sont rares et peu d'informations sont disponibles. Ainsi, s'il est généralement admis que les filaments d'actine se polymérisent autour des points d'adhésion, puis que les fibres de stress s'assemblent entre ces points, nous ne connaissons pas de description concernant l'implication réelle des microtubules. Même si l'on sait qu'ils se polymérisent depuis le centrosome vers les points d'adhésion, rien ne permet de dire si ce processus a lieu en amont, en parallèle ou après la création des filaments d'actine et des fibres de stress. Ainsi, pour palier ce manque d'informations, un scénario simple d'adhésion et d'étalement cellulaire peut être considéré : lorsque la cellule perçoit le substrat à une certaine distance, des microtubules se polymérisent à partir du centrosome et en direction des molécules d'adhésion, qui sont ainsi poussées vers le substrat jusqu'à leur adhésion. Ce processus se reproduit progressivement, jusqu'à étalement complet de la cellule [22].

Une première série de résultats issue de cette simulation est présentée pour le cas d'une cellule dont le centrosome se situe à l'aplomb du noyau. Le réseau de filaments en tension ainsi obtenu apparaît sur la figure 3 (vue de côté à gauche, vue de dessus au centre). On voit que les éléments les plus tendus sont situés en face basale, certains étant sur les côtés et joignant les points d'adhésion, tandis que les moins tendus sont plutôt en face apicale. Ceci est cohérent avec la plupart des observations in vivo menées sur les répartitions des fibres de stress et de l'actine corticale (Fig. 3, droite) [3, 11].

La figure 4 présente à présent le réseau de filaments en compression. On note qu'ils rayonnent à partir du centrosome vers les intégrines (distribution dite étoilée), ce qui également en adéquation avec des observations expérimentales, dont un exemple est montré sur la droite de la figure 3 [23]. La simulation dévoile par ailleurs que 

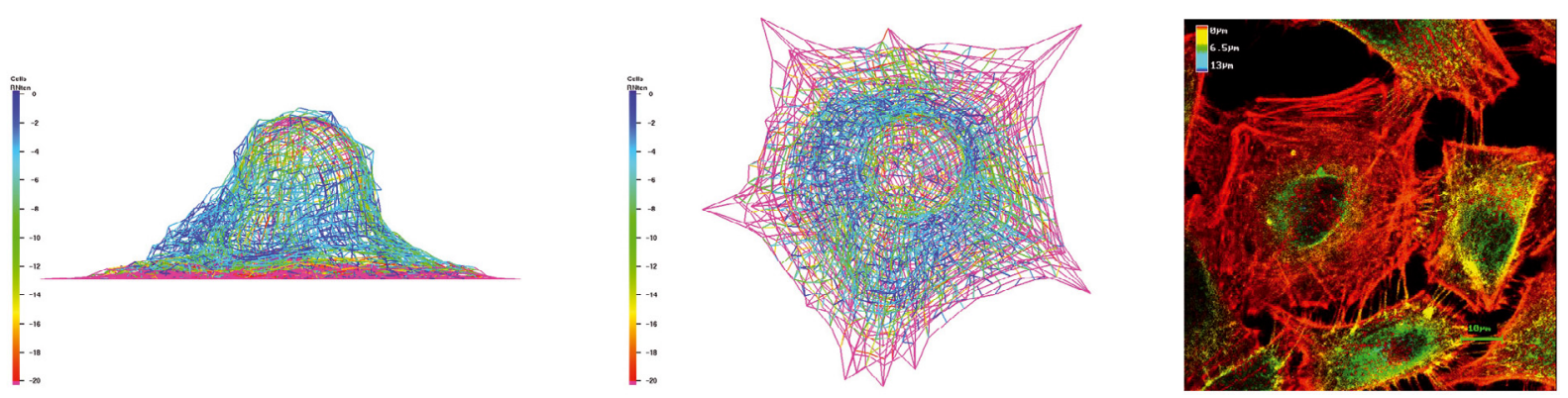

Fig. 3. (a) et (b) Réseau en tension de microfilaments, (c) observation expérimentale.
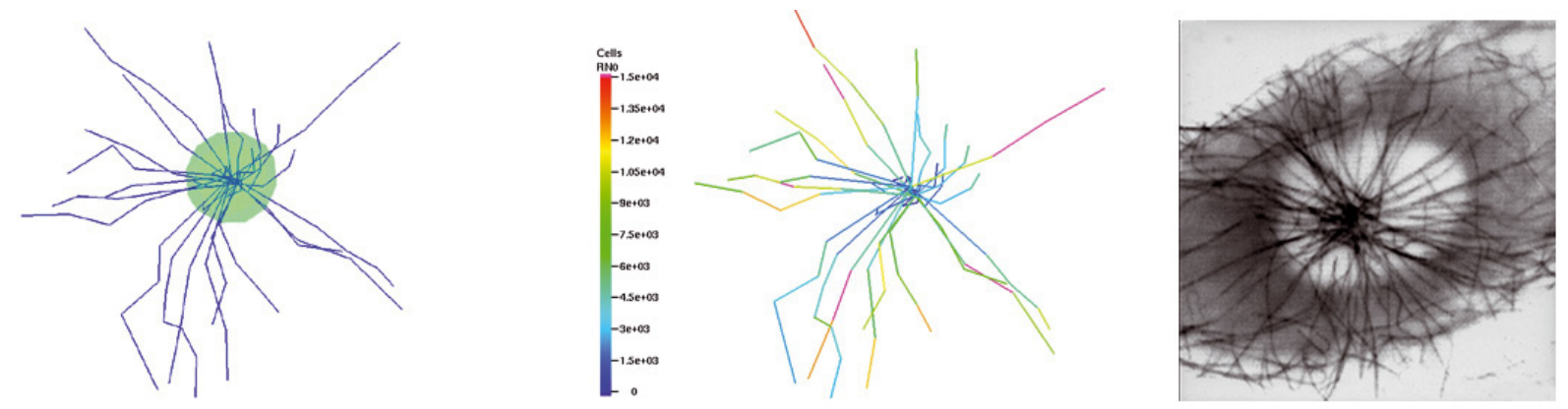

Fig. 4. Réseau en compression de microtubules et observation expérimentale.

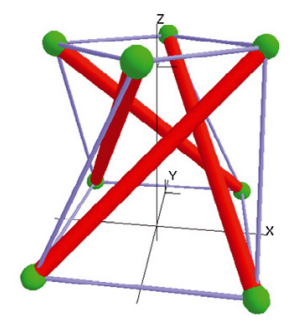

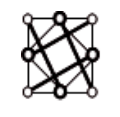

1

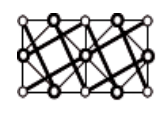

2

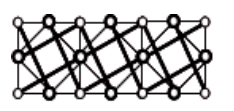

4

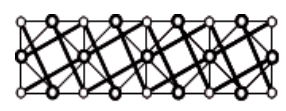

6

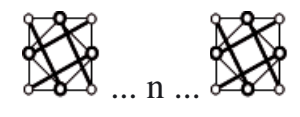

$2(n-1)$

Fig. 5. Évolution du nombre d'états d'autocontrainte en fonction du nombre de cellules.

le niveau de compression dans les microtubules décroit au long du processus d'adhésion. Cela traduit en fait le transfert progressif de ces efforts vers le substrat grâce à l'accroissement du taux d'adhésion, selon un phénomène déjà observé [14].

\section{De la cellule vers le tissu : le potentiel des grilles de tenségrité}

L'étape suivante, qui reste néanmoins à franchir, vise à l'extension de la modélisation mécanique par systèmes de tenségrité au cas des complexes multicellulaires. Cette approche est basée sur la propriété des systèmes de tenségrité de pouvoir s'associer pour former des structures organisées et complexes. Ces assemblages présentent la caractéristique importante de présenter des contraintes communes à un sous-groupe de cellules tout en conservant l'individualité de chaque élément [24].

Cet aspect est illustré sur la figure 5, où l'assemblage successif de systèmes de quadruplex fait apparaître un nombre d'état d'autocontrainte supérieur au nombre de quadruplex. Par exemple, pour quatre systèmes alignés, il apparaît six états : quatre sont propres à chaque quadruplex et deux sont dits «partagés » car ils impliquent des éléments appartenant à différents systèmes.

On montre pareillement qu'un assemblage planaire de six par six quadruplex fait apparaître 124 états d'autocontrainte, dont 36 liés à chaque système et 88 partagés entre différents quadruplex (un exemple de ces états est présenté en Fig. 6).

L'intérêt sur le plan mécanique et biologique est manifeste. Cela permet d'identifier des chemins privilégiés de l'autocontrainte, alors considérée comme un partage d'information entre les cellules d'un groupement, et d'en déduire un mode de transmission des efforts entre cellesci, notamment l'existence de chemins spécifiques. Par exemple, si un élément de câble se rompt sur une cellule, l'ensemble de celle-ci est affecté (disparition de son état d'autocontrainte, signifiant éventuellement sa mort), mais cela va également se répercuter sur d'autres cellules du tissu, même non adjacentes et éloignées, à partir du moment où elles sont impliquées dans un état d'autocontrainte partagée avec le câble déficient de cette cellule. 


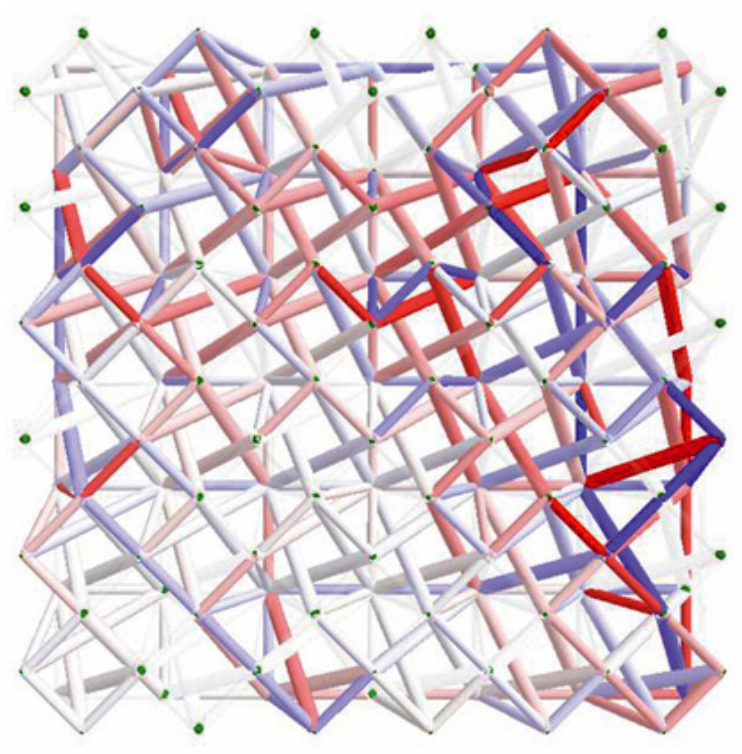

Fig. 6. Etat d'autocontrainte partagé entre $6 \times 6$ quadruplex.

Sur un plus long terme, ces approches devraient pouvoir contribuer à l'identification des modes de propagation des contraintes et des déformations entre cellules jointives constitutives d'un tissu, mettant ainsi en évidence des situations relevant de la contribution d'une seule cellule ou de celle d'un sous-groupe cellulaire. Ces résultats visent à mieux appréhender l'organisation d'un tissu afin de caractériser plus pertinemment sa réponse mécanique sous sollicitation externe, question plus que jamais d'actualité dans les besoins et recherches en ingénierie tissulaire.

\section{Conclusion}

Une meilleure compréhension de nombreux mécanismes biologiques nécessite de disposer de méthodes et outils de modélisation dédiés à l'étude du comportement mécanique du cytosquelette cellulaire. Les systèmes de tenségrité ont démontré leur pertinence et efficacité à cet égard. Ils sont en effet aptes à représenter de façon bio-réaliste l'architecture complexe du cytosquelette ainsi que la répartition non régulière des forces de tension et de compression en son sein. Associés aux outils des milieux divisés, ils offrent aussi la possibilité de modéliser son processus de structuration lors de l'adhésion cellulaire. En outre, ils ont des propriétés d'assemblage permettant de générer des modèles de tissus permettant une approche multi-échelles, depuis le niveau de filaments du cytosquelette jusqu'au niveau tissulaire.

Les résultats issus des simulations numériques menées à partir de l'analogie avec les systèmes de tenségrité sont validés par la plupart des observations expérimentales, ce qui traduit le potentiel de cette approche et laisse présager de futures avancées.

\section{Références}

[1] S. Kumar, I.Z. Maxwell, A. Heisterkamp, T.R. Polte, T. Lele, M. Salanga, E. Mazur, D.E. Ingber, Viscoelastic retraction of single living stress fibers and its impact on cell shape, cytoskeletal organization and extracellular matrix mechanics, Biophys. J. 90 (2006) 3762-3773

[2] B. Fabry, G. Maksym, J.P. Butler, M. Glogauer, D. Navajas, J.J. Fredberg, Scaling the microrheology of living cells. Phys. Rev. Lett. 87 (2001) 148102

[3] V.M. Laurent, R. Fodil, P. Cañadas, S. Fereol, B. Louis, E. Planus, D. Isabey, Partitioning of cortical and deep cytoskeleton responses from transient magnetic bead twisting. Ann. Biomed. Eng. 31 (2003) 1263-1278

[4] M. Puig-de-Morales, E.J. Millet, B. Fabry, D. Navajas, N. Wang, J.P. Butler, J.J. Fredberg, Cytoskeletal mechanics in adherent human airway smooth muscle cells : probe specificity and scaling of protein-protein dynamics. Am. J. Physiol. : Cell Phys. 287 (2004) 643-654

[5] A.K. Harris, R. Wild, and D. Stopak, Silicone rubber substrata : a new wrinkle in the study of cell locomotion, Science 208 (1980) 177-179

[6] D. Stamenovic, S. Mijailovic, I. Tolic-Norrelykke, J. Chen, N. Wang, Cell pre-stress II. Contribution of microtubules. Am. J. Cell Physiol. 282 (2002) 617-624

[7] C.P. Brangwynne, F.C. MacKintosh, S. Kumar, N.A. Geisse, J. Talbot, L. Mahadevan, K.K. Parker, D.E. Ingber, D.A. Weitz, Microtubules can bear enhanced compressive loads in living cells because of lateral reinforcement. J. Cell Biol. 173 (2006) 733-741

[8] D. Stamenovic, M.F. Coughlin, The role of prestress and architecture of the cytoskeleton and deformability of cytoskeletal filaments in mechanics of adherent cells : a quantitative analysis. J. Theor. Biol. 201 (1999) 63-74

[9] P. Cañadas, V.M. Laurent, P. Chabrand, D. Isabey, and S. Wendling-Mansuy, Mechanisms Governing the ViscoElastic Responses of Living Cells Assessed by Foam and Tensegrity Models, Med. Biol. Eng. Comput. 416 (2003) 733-739

[10] D. Stamenovic, D.E. Ingber, N. Wang, and J.J. Fredberg, A Microstructural Approach to Cytoskeletal Mechanics Based on Tensegrity, J. Theor. Biol. 181 (1996) 125-136

[11] S. Wendling, C. Oddou, and D. Isabey, Stiffening Response of a Cellular Tensegrity Model, J. Theor. Biol. 1963 (1999) 309-325

[12] D.E., Ingber, Cellular tensegrity : defining new rules of biological design that govern the cytoskeleton, J. Cell Sci., 104 (1993) 613-627

[13] N. Wang, K. Naruse, D. Stamenovic, J.J. Fredberg, S.M. Mijailovich, I.M. Tolic-Norrelykke, T. Polte, R. Mannix, D.E. Ingber, Mechanical behavior in living cells consistent with the tensegrity model, Proceedings of the National Academy of Sciences of the USA 98, Vol. 14 (2001) 77657770

[14] S. Wendling, P. Cañadas, C. Oddou, and A. Meunier, Interrelations between elastic energy and strain in a tensegrity model ; contribution to the analysis on the mechanical response in living cells, Comput. Methods Biomech. Biomed. Eng. 5 (2002) 1-6

[15] S. Wendling, P. Cañadas, and P. Chabrand, Toward a generalized tensegrity model describing the mechanical behaviour of the cytoskeleton structure, Cornput. Methods Biornech. Biorned. Eng. 1 (2003) 1-8 
[16] C. Sultan, D. Stamenovic, D.E. Ingber, A computational tensegrity model predicts dynamic rheological behaviors in living cells, Annals Biomed. Eng. 32 (2004) 520-530

[17] P. Cañadas, V.M. Laurent, C. Oddou, D. Isabey, and S. Wendling, A Cellular Tensegrity Model to Analyse the Structural Viscoelasticity of the Cytoskeleton, J. Theor. Biol. 218 (2002) 155-173

[18] P. Cañadas, S. Wendling-Mansuy, D. Isabey, Frequency response of a viscoelastic trensegrity structure : structural rearrangement contribution to cell dynamics, ASME J. Biomech. Eng. 128 (2006) 487-495

[19] L. Zhang, B. Maurin, R. Motro, Form-finding of non regular tensegrity systems, Journal of Structural Engineering 132 (2006) 1435-1440

[20] H. Baudriller, B. Maurin, P. Cañadas, P. Montcourrier, A. Parmeggiani, N. Bettache, Form-finding of complex tensegrity structures application to cell cytoskeleton modelling Comptes Rendus de l'Académie des Sciences Mécanique 334 (2006) 662-668
[21] J.L. Milan, S. Wendling-Mansuy, M. Jean, P. Chabrand, Divided medium-based model for analyzing the dynamic reorganization of the cytoskeleton during cell deformation, Biomechan. Model Mechanobiol. 6 (2007) 373-390

[22] B. Maurin, P. Cañadas, H. Baudriller, P. Montcourrier, N. Bettache. Mechanical model of cytoskeleton structuration during cell adhesion and spreading, Journal of Biomechanics 41 (2008) 2036-2041

[23] TJ. Keating, J.G. Peloquin, V.I. Rodionov, D. Momcilovic, G.G. Borisy, Microtubule release from de centrosome, Proceedings of the National Academy of Sciences of the USA 94 (1997) 5078-5083

[24] L. Sanchez-Sandoval, B. Maurin, M.N. Kazi Aoual, R. Motro, Selfstress states identification and localization in modular tensegrity grids, International Journal of Space Structures 22 (2007) 215-224 